\title{
Patient Diet Recommendation System Using K Clique and Deep learning Classifiers
}

\author{
Dr. Samuel Manoharan, \\ Professor, \\ Department of Electronics, \\ Bharathiyar College of Engineering and Technology, \\ India. \\ Email: jsamuel@bcetedu.in \\ Prof. Sathish, \\ Department of EEE, \\ Eritrea Institute of Technology, \\ Eritrea. \\ Email: sathesh4you@gmail.com
}

\begin{abstract}
There are several systems designed for the purpose of recommending. The recommending system has gained its prominence even in the medical industry for suggesting the diets for the patient's, medicines to be taken, treatments to be taken etc. The recommendation system mainly enhances the robustness, extends protection against the many disease and improves the quality of living of an individual. So to automatically suggest the foods based on their health conditions and the level of sugar, blood pressure, protein, fat, cholesterol, age etc. the paper puts forth k-clique embedded deep learning classifier recommendation system for suggesting the diets for the patients. The K-clique incorporated in the recommendation system in an effort of getting an improved preciseness and increasing the accuracy of the deep learning classifier (gated recurrent units). The dataset for the empirical analysis of the developed system was performed with the data set of the patients collected over the internet as well as hospitals, information's of about 50 patients were collected with thirteen features of various disease and thousand products with eight feature set. All these features were encoded and grouped into several clusters before applying into the deep learning classifiers. The better preciseness and the accuracy observed for the developed system experimentally is compared with the machine learning techniques such as logistic regression and Naïve Bayes and other deep learning classifiers such as the MLP and RNN to demonstrate the proficiency of the K-clique deep learning classifier based recommendation system (K-DLRS)
\end{abstract}

Keywords: Patient Recommendation System, K-clique, Deep Learning Classifiers, Gated Recurrent Units, K-DLRS, Improved Preciseness and Accuracy.

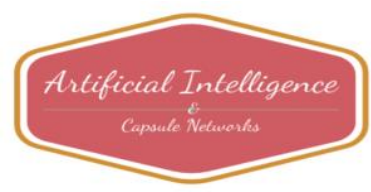


Journal of Artificial Intelligence and Capsule Networks (2020)

Vol.02/ No. 02

Pages: 121-130

http://irojournals.com/aicn/

DOI: https://doi.org/10.36548/jaicn.2020.2.005

\section{Introduction}

The recommending systems for the patients diets tracks the complete health conditions of the patients in a personalized manner and recommends the suitable and the variety of the food items that the patients can have. The dieting recommending system (DRS) is carefully utilized to provide the suggestion on the diets that could be used as a nutritional supplement to the patients as well as the food products that satisfies the health requisites, tastes and the dietary preferences. Further the on the basis of the healthy living the DRS are capable of delivering optimal solutions that would meet the patients choices on foods taking into consideration the huge amount of data accessible and correlated to foods or the recipes.

Manifold procedures and algorithms offering an efficient suggestions have been put forth by many researchers. This is presented in the related work section of the paper. The researches conducted so far has demonstrated that the diet suggestion provided to the patients were indeed a medication to the many patients who have diseases. In the DRS centered product details with the automated suggestion, food habits as suggested by the dietitians based on the health conditions would enhance the robustness, extending protection against the many disease and improving the quality of living of an individual.

The nutritious foods improves the health condition by providing a proper level of vitamins, antioxidants, minerals, proteins, fiber and the fat that are essential for the body they also remain as the fundamental factor to the perfect physical functioning of the body and sometimes causes defects in the patient's body that are allergic to the nutritious intake because of the disease they are suffering from.

The real time suggestion of healthy system for the patients with regard to the nutritional requisite of the patients has become a very serious issue for the researchers. So the proposed method in the paper puts forth an DRS with the deep learning classifier to develop an automatic food suggestion RS and further embeds the cosine similarity and the K-Clique into the DRS along with the deep learning models to identify the similar patients with same disorders, who require a same type of diet and group them respectively. Incorporating the K-clique in the DRS along with the DL- Model enhances the preciseness and the accuracy of the deep learning techniques.

The remaining of the paper proceeds with the section 2 presenting the related works about the diverse applications of the recommendation system, the section 3 providing the proposed method on the developing a K-DLRS for the diet suggestion based on the patients' needs and disorders. Section 4 with the empirical observation of the developed system and comparison with the other models. And section 5 the conclusion of the work.

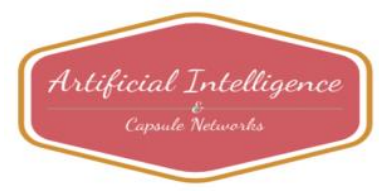


Journal of Artificial Intelligence and Capsule Networks (2020)

Vol.02/ No. 02

Pages: 121-130

http://irojournals.com/aicn/

DOI: https://doi.org/10.36548/jaicn.2020.2.005

\section{Related works}

Phanich, et al [1] as nutritious diets are the important for maintaining a good health, it becomes necessary for everyone to intake a healthy diet. The paper devises a recommendation system that suggests a nutrition therapy as a key remedy for the diabetic patients who have diverse food restrictions. The author utilizes the clustering analysis method and the Self organizing method to formulate a FRS for the diabetes patients to provide perfect suggestion on their food intake.

Jeong, et al [2] demonstrates the applicability of the recommendation system to the movie lens, the proposed methodology of the paper utilizes the "personal propensity and the secure collaborating filtering to deliver remedies to the sparsity and the scalability problems".

Hao, et al [3] scopes in surveying the various social network analysis strategies to examine the social frame work and the significant properties using the network and the graph theories, utilizing the "soft computing strategies like the fuzzy logic the formal concept examination using the rough set theory and the soft set theory".

Tran et al [4] the increasing attention of the FRS because of their significance in the healthy living is the motivation behind this paper is to present the comprehensive over view of the FRS for the individuals as well as cluster of people using the healthy food domain. The paper also examines the current standard FRS, and presents the discussion on the research issues associated in designing a next generation suggestion technologies. Norouzi et al [5] Smart FRS developed in the paper uses the artificial intelligence to design a knowledge base according to the guiding principle laid out by the "American diabetes association". The favorites and the health status of the patients was analyzed to suggest a perfect snack for the affected.

Xinchang, et al [6] presents the "Movie Recommendation Algorithm Using Social Network Analysis to Alleviate Cold-Start Problem."Vilakone et al [7] proposes "The Efficiency of a DoParallel Algorithm and an FCA Network Graph Applied to Recommendation System."Vilakone, et al [8] put forth a "Personalized Movie Recommendation System Combining Data Mining with the k-Clique Method."

Smys, S et al [9] put forth the. "Big Data Business Analytics as a Strategic Asset for Health Care Industry."Pandian, et al [10] devised a "Sleep Pattern Analysis and Improvement Using Artificial Intelligence and Music Therapy." Bashar et al [11] presents the. "Survey on Evolving Deep Learning Neural Network Architectures." Manoharan, et al [12] presents the feature detection based on the "Hermitian graph

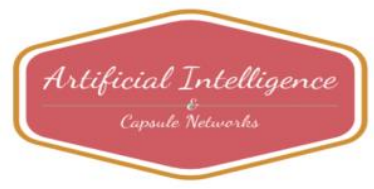


Journal of Artificial Intelligence and Capsule Networks (2020)

Vol.02/ No. 02

Pages: 121-130

http://irojournals.com/aicn/

DOI: https://doi.org/10.36548/jaicn.2020.2.005

wavelets." Based some of the above mentioned methodologies the paper devises an automated DRS, the system relies on the deep learning method, encoding the features before classification, moreover the model with the deep learning includes the cosine similarity algorithm put forth in "An efficient similarity join algorithm with cosine similarity predicate" by Lee et al [13] and the K-clique put forth by Duan, et al [14] in "Incremental K-clique clustering in dynamic social networks." To identify the similar patients with similar disorder and the dietary product and cluster them to increase the accuracy in the deep learning. The outcome of the developed system is compared with the other deep learning models such as RNN, MLP etc [15] used in the FRS and machine learning models such as the logistic regression and the Naïve Bayes. The deep learning model used in the proposed DRS is GRU- Gated Recurrent Unit that was utilized in health monitoring application put forth by Zhao et al [16]

\section{K-DLRS for Diet Recommendations}

The paper scopes in developing a DRS to suggest the diet for the diverse patient with different type of disease automatically. The gated recurrent unit used in the paper learns the features of the medical dataset gathered based on the disorders found in the patient, and other information's based on their health condition, age and the gender. The data set with the 50 patients details their disorders, heath status and the other particulars mentioned above are gathered over internet and totally more than thousand products were tested over patients with different disease, the data set was comprised with eight product features and thirteen patient features and an average of 17,000 records. The table.1 below list the nutritional facts of the products and health status of the patients.

\begin{tabular}{|l|l|}
\hline \multicolumn{1}{|c|}{$\begin{array}{c}\text { Food Product Features } \\
\text { Code of The Product }\end{array}$} & Number \\
\hline $\begin{array}{l}\text { Calories in Product } \\
\text { Proteins in Product }\end{array}$ & $\begin{array}{l}\text { Age } \\
\text { Gender }\end{array}$ \\
\hline $\begin{array}{l}\text { Fat in Product } \\
\text { Sodium in Product }\end{array}$ & Disorder \\
\hline Carbohydrate in Product & Level of Disorder \\
\hline Fiber in Product & $\begin{array}{l}\text { Health Condition (fat, } \\
\text { cholesterol, weight ) }\end{array}$ \\
\hline Cholesterol in Product & $\begin{array}{l}\text { Level of minerals in body } \\
\text { (protein, sodium, fiber, } \\
\text { calories) }\end{array}$ \\
\hline
\end{tabular}

Table.1 Product and Patient Features

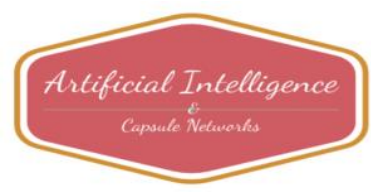


Journal of Artificial Intelligence and Capsule Networks (2020)

Vol.02/ No. 02

Pages: 121-130

http://irojournals.com/aicn/

DOI: https://doi.org/10.36548/jaicn.2020.2.005

The data set is preprocessed by removing the noises, from the data set and normalizing its features. The normalization scales the dataset to single range as the dataset features are in various scales. The proposed method uses the min-max scaling to normalize the values between 0 and 1 using the equation (1) shown below

$$
\text { normalization }(i)=\frac{\text { Feature }(i)-\min (\text { Feature })}{\max (\text { Features })-\operatorname{Min}(\text { Features })}
$$

The data is converted into numeric value for easy processing and encoded. The similarities in the data set is identified by applying the cosine similarity algorithm and clustered based on the adjacency matrix using the K-clique. The figure. 1 below shows the proposed flow diagram. The food product and the people with similar disorders are grouped and the model is trained with the appropriate food products for the people with disorders.

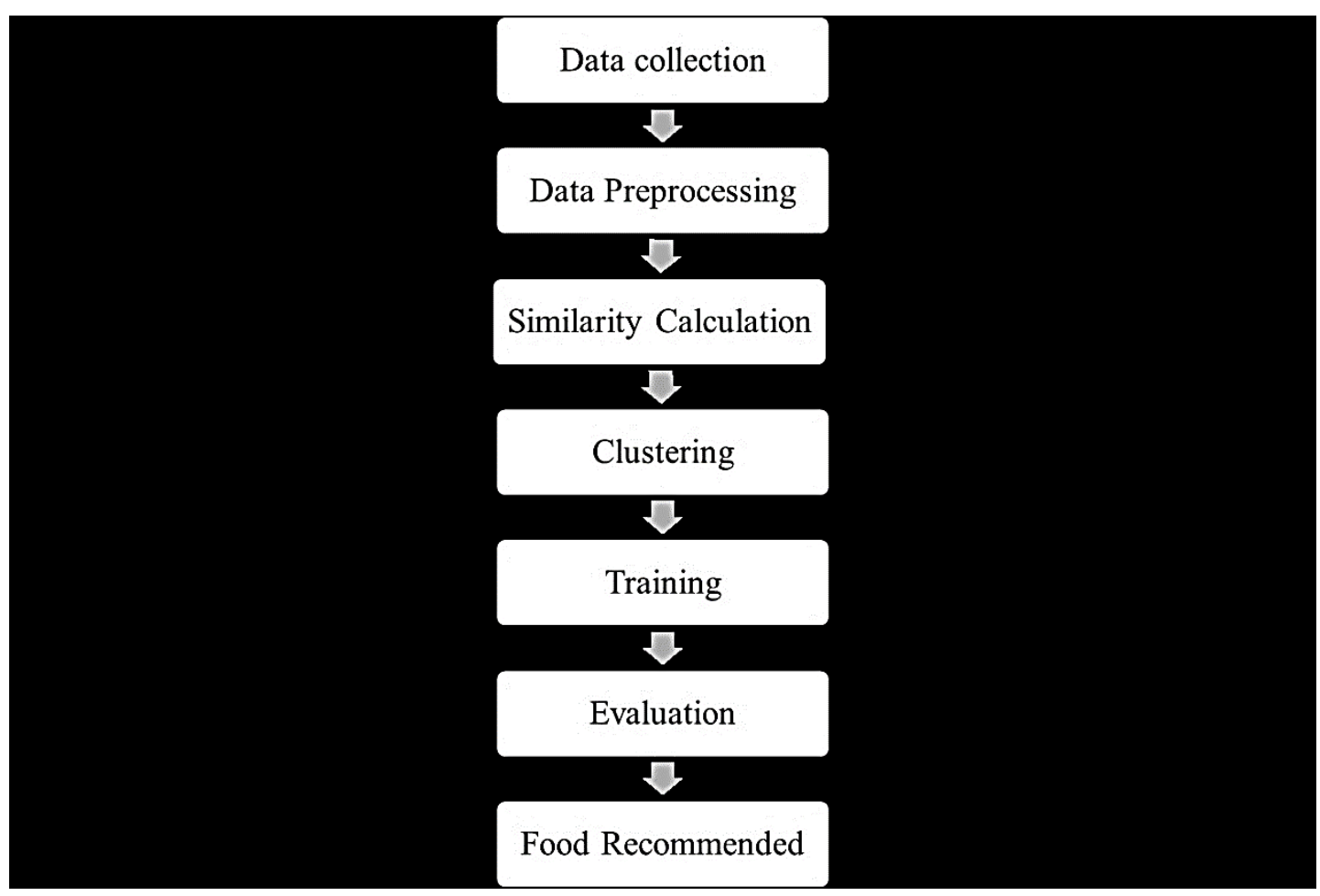

Figure.1 Proposed Flow Diagram

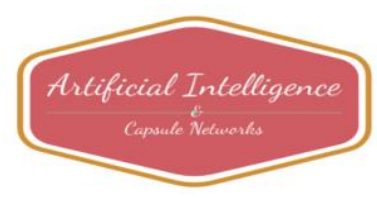


Journal of Artificial Intelligence and Capsule Networks (2020)

Vol.02/ No. 02

Pages: 121-130

http://irojournals.com/aicn/

DOI: https://doi.org/10.36548/jaicn.2020.2.005

Based on the grouped features the model is trained using the gated recurrent units that does fastest and efficient training. The trained model is now ready to suggest the diet for the patients based on the training provided to the model. The empirical results observed in the next section proves the efficiency of the model developed.

\section{Performance Evaluation}

The developed model is designed by evaluating the using the dataset collected from over the internet. The paper uses the performance metric accuracy, precision, recall and f- measure for benchmarking the outcome of the proposed model. The outcomes are achieved over the core i5 processor with 128 GB RAM for about 50 Epochs. The dataset is categorized into training set, testing and cross validation dataset a Kfold-cross validation is utilized for both training and testing.

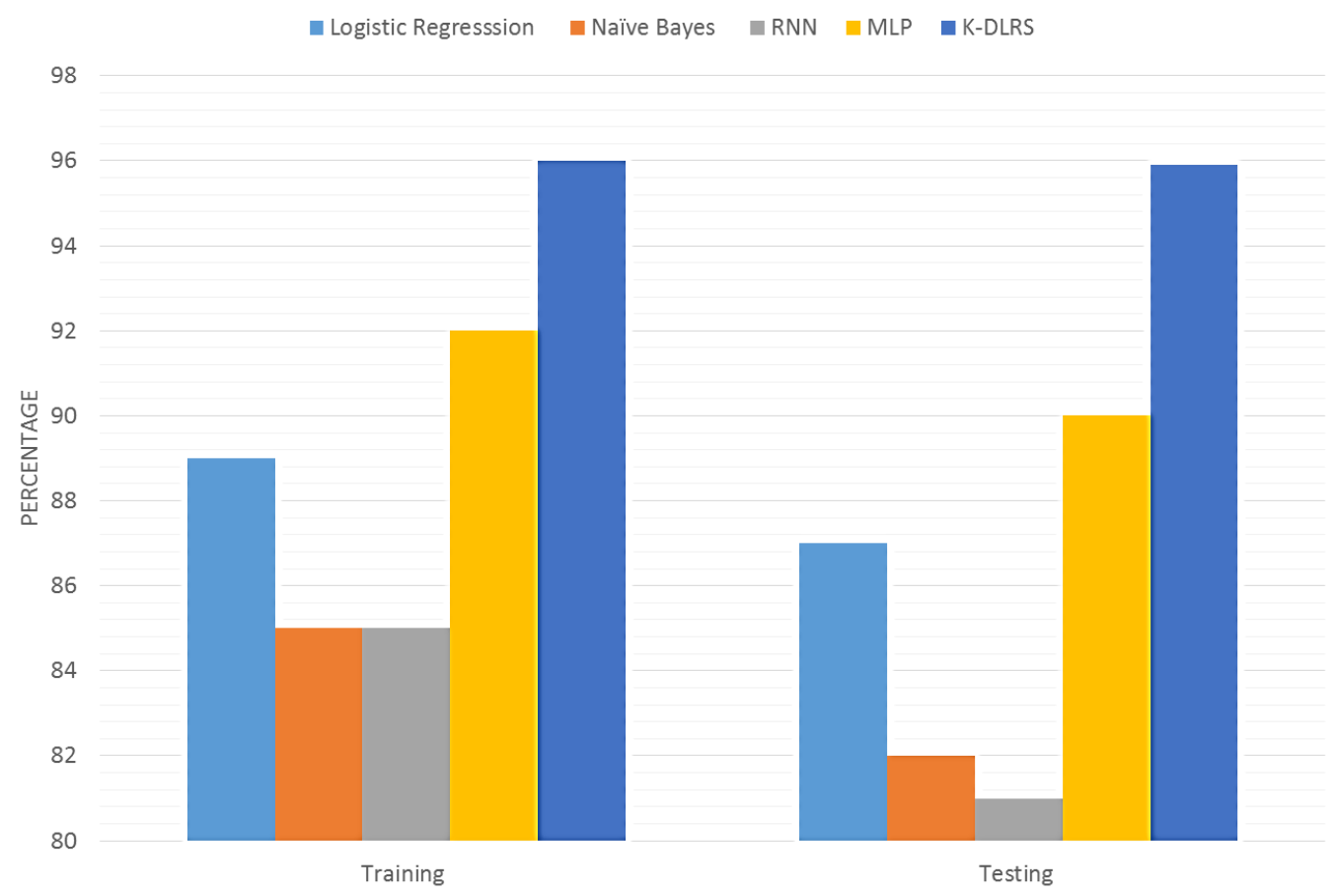

Figure. 2 Training and Testing Accuracy

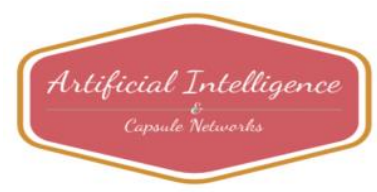


Journal of Artificial Intelligence and Capsule Networks (2020)

Vol.02/ No. 02

Pages: 121-130

http://irojournals.com/aicn/

DOI: https://doi.org/10.36548/jaicn.2020.2.005

The figure. 2 is the training and the testing accuracy of the different machine learning logistic regression and Naïve Bayes and the diverse individual deep learning models based DRS such as RNN MLP etc., as well as proposed K-DLRS the outcome shows that the system developed in the paper is highly precise and accurate than the other models.

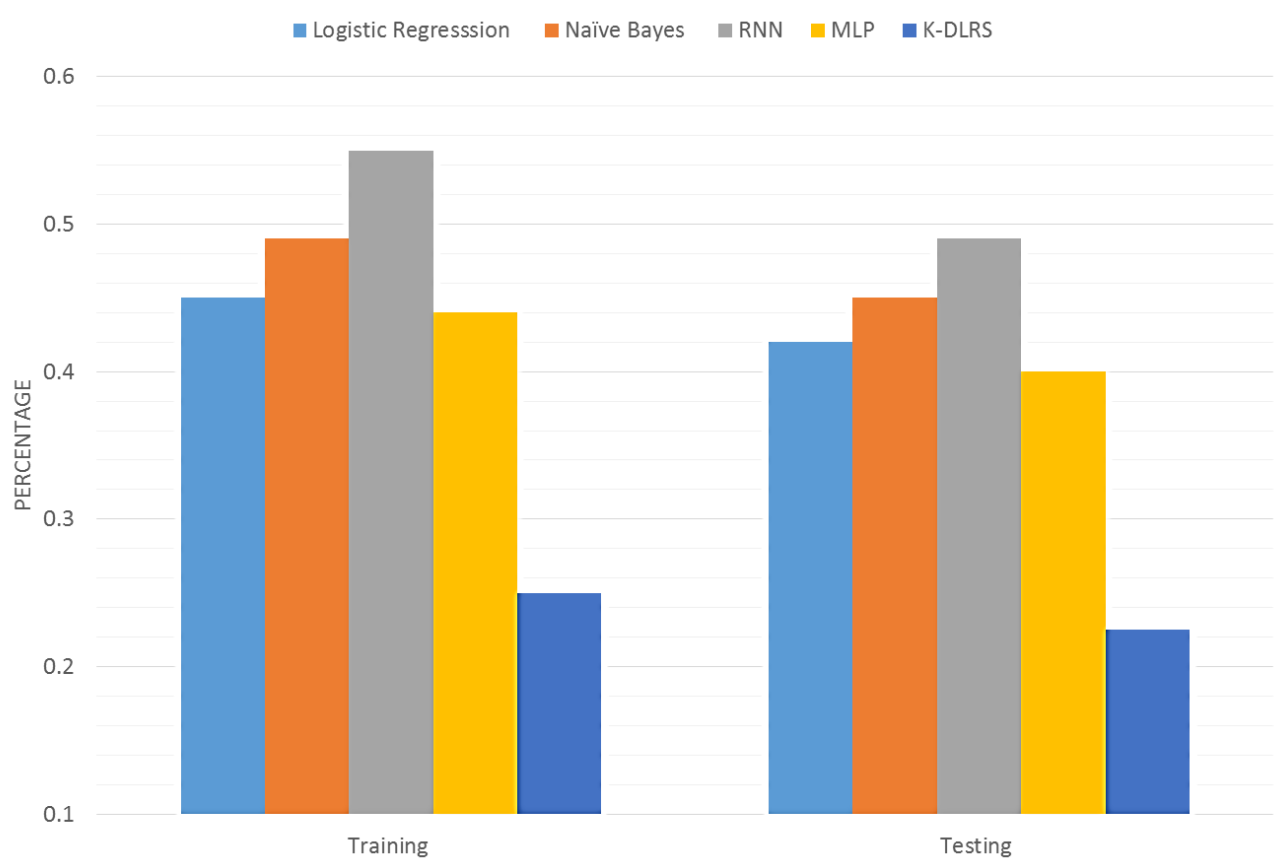

Figure.3 Training and Testing Loss

The figure. 3 is the training and the testing loss of the different machine learning logistic regression and Naïve Bayes and the diverse individual deep learning models based DRS such as RNN MLP etc., as well as proposed K-DLRS the outcome shows that the system developed in the paper has a reduced loss compared to the other models.

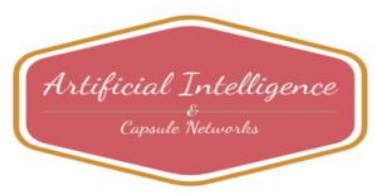


Journal of Artificial Intelligence and Capsule Networks (2020)

Vol.02/ No. 02

Pages: 121-130

http://irojournals.com/aicn/

DOI: https://doi.org/10.36548/jaicn.2020.2.005

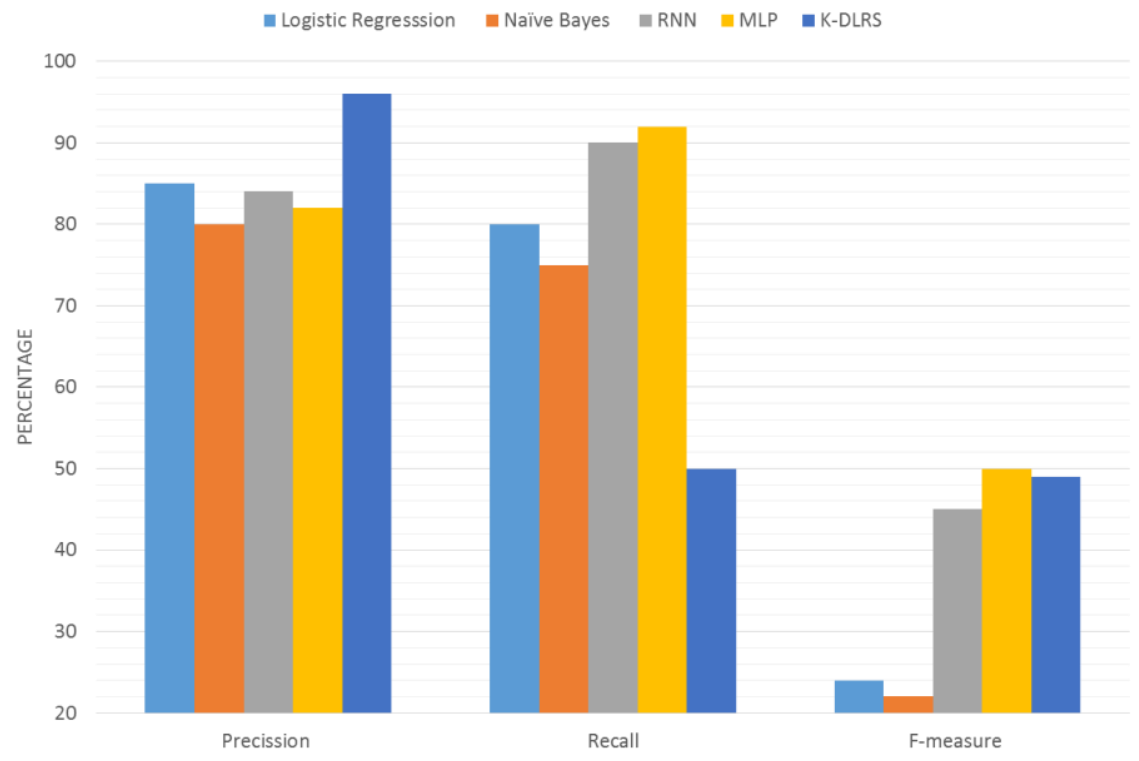

Figure.4 Classification Results (F-measure, Precision and Recall)

The Figure. 4 shows the classification results for the proposed model along with the other machine learning and the deep learning models. The outcome observed from the experiments demonstrates the competencies of the K-DLRS in terms of precision recall and the F-measure. The accuracy, precision, Recall and the Fmeasure for the models are calculated based on the true positive, false positive, true negative and the false negative observed.

\section{Conclusion}

The recommendation system for dietary food based on the disorders, the health conditions and the other features of the patients is developed in the system using the K-clique and the gated recurrent network. Based on the dataset collected over the internet. The features of the products and the patients are sorted out, preprocessed, and encoded and segregated based on the similarities and used for training the model. The model is trained fast using the gated recurrent network. The developed design is trained, tested and cross validated. The outcomes of the training, testing and cross validating demonstrates that the proposed system has better precise and accuracy than the other ML and DL procedures such as the MLP, RNN and Logistic Regression, Navies' Bayes respectively.

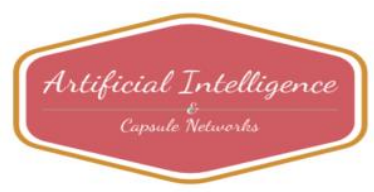


Journal of Artificial Intelligence and Capsule Networks (2020)

Vol.02/ No. 02

Pages: 121-130

http://irojournals.com/aicn/

DOI: https://doi.org/10.36548/jaicn.2020.2.005

\section{References}

[1] Phanich, Maiyaporn, Phathrajarin Pholkul, and Suphakant Phimoltares. "Food recommendation system using clustering analysis for diabetic patients." In 2010 International Conference on Information Science and Applications, pp. 1-8. IEEE, 2010.

[2] Jeong, Woon-hae, Se-jun Kim, Doo-soon Park, and Jin Kwak. "Performance improvement of a movie recommendation system based on personal propensity and secure collaborative filtering." Journal of Information Processing Systems 9, no. 1 (2013): 157-172

[3] Hao, Fei, Doo-Soon Park, and Zheng Pei. "When social computing meets soft computing: opportunities and insights." Human-centric Computing and Information Sciences 8, no. 1 (2018)

[4] Tran, Thi Ngoc Trang, Müslüm Atas, Alexander Felfernig, and Martin Stettinger. "An overview of recommender systems in the healthy food domain." Journal of Intelligent Information Systems 50, no. 3 (2018): 501-526.

[5] Norouzi, Somaye, Azade Kamel Ghalibaf, Samane Sistani, Vahideh Banazadeh, Fateme Keykhaei, Parisa Zareishargh, Fateme Amiri, Mohsen Nematy, and Kobra Etminani. "A Mobile Application for Managing Diabetic Patients' Nutrition: A Food Recommender System." Archives of Iranian Medicine (AIM) 21, no. 10 (2018).

[6] Xinchang, Khamphaphone, Phonexay Vilakone, and Doo-Soon Park. "Movie Recommendation Algorithm Using Social Network Analysis to Alleviate Cold-Start Problem." Journal of Information Processing Systems 15, no. 3 (2019).

[7] Vilakone, Phonexay, Khamphaphone Xinchang, and Doo-Soon Park. "Personalized Movie Recommendation System Combining Data Mining with the k-Clique Method." Journal of Information Processing Systems 15, no. 5 (2019).

[8] Vilakone, Phonexay, and Doo-Soon Park. "The Efficiency of a DoParallel Algorithm and an FCA Network Graph Applied to Recommendation System." Applied Sciences 10, no. 8 (2020): 2939.

[9] Smys, S., and C. V. Joe. "Big Data Business Analytics as A Strategic Asset For Health Care Industry." Journal of ISMAC 1, no. 02 (2019): 92-100.

[10] Pandian, M. Durai. "Sleep Pattern Analysis and Improvement Using Artificial Intelligence and Music Therapy." Journal of Artificial Intelligence 1, no. 02 (2019): 54-62.

[11] Bashar, Abul. "Survey on Evolving Deep Learning Neural Network Architectures." Journal of Artificial Intelligence 1, no. 02 (2019): 73-82.

[12] Manoharan, Samuel. "Study On Hermitian Graph Wavelets in Feature Detection." Journal of Soft Computing Paradigm (JSCP) 1, no. 01 (2019): 24-32.

[13] Lee, Dongjoo, Jaehui Park, Junho Shim, and Sang-goo Lee. "An efficient similarity join algorithm with cosine similarity predicate." In International Conference on Database and Expert Systems Applications, pp. 422-436. Springer, Berlin, Heidelberg, 2010.

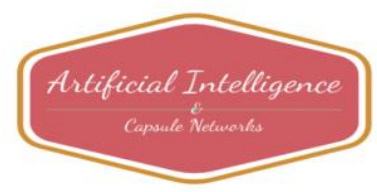


Journal of Artificial Intelligence and Capsule Networks (2020)

Vol.02/ No. 02

Pages: 121-130

http://irojournals.com/aicn/

DOI: https://doi.org/10.36548/jaicn.2020.2.005

[14] Duan, Dongsheng, Yuhua Li, Ruixuan Li, and Zhengding Lu. "Incremental K-clique clustering in dynamic social networks." Artificial Intelligence Review 38, no. 2 (2012): 129-147

[15] Wei, Jian, Jianhua He, Kai Chen, Yi Zhou, and Zuoyin Tang. "Collaborative filtering and deep learning based recommendation system for cold start items." Expert Systems with Applications 69 (2017): 29-39.

[16] Zhao, Rui, Dongzhe Wang, Ruqiang Yan, Kezhi Mao, Fei Shen, and Jinjiang Wang. "Machine health monitoring using local feature-based gated recurrent unit networks." IEEE Transactions on Industrial Electronics 65, no. 2 (2017): 1539-1548.

\section{Authors Biography}

Dr. Samuel Manoharan, is a professor in Department of Electronics, in Bharathiyar College of Engineering and Technology, India. His area of research are Artificial Intelligence, Robotics, Deep Learning, Cognitive Science, Ad-hoc Networks, Artificial Neural Network, Evolutionary Computing, Speech Recognition and Autonomous Systems

Prof .Sathish works in the Department of EEE,in Eritrea Institute of Technology,Eritrea. His interested area of research includes modern computer Science and emerging smart technologies in various aspects of Wireless Communications, Cloud Computing, Computer System Engineering, Communication Technologies, Information Processing, Computer Networks, Web Technologies, Computing \& Communications, Automation, Image processing and Wireless Communications.

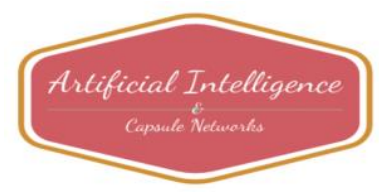

